\title{
Follicular Dowling-Degos Disease with Hidradenitis Suppurativa: A Case Report and Review of the Literature
}

\author{
Ya-Nin Nokdhes Thanachat Rutnumnoi Poramin Patthamalai \\ Charussri Leeyaphan \\ Department of Dermatology, Siriraj Hospital, Faculty of Medicine, Mahidol University, \\ Bangkok, Thailand
}

\section{Keywords}

Follicular Dowling-Degos disease $\cdot$ Hidradenitis suppurativa $\cdot$ Acne

\begin{abstract}
Dowling-Degos disease (DDD) is an autosomal dominant disorder with variable phenotypic expression. Classically, DDD is characterized by progressive reticulate hyperpigmentation on flexures with perioral pitted scars and comedone-like hyperkeratotic papules. Follicular DDD is a rare variant which was introduced by Singh et al. [Indian J Dermatol Venereol Leprol. 2013 Nov-Dec;79(6):802-4]. Follicular DDD differs from other variants because of its notable comedone-like hyperkeratotic hyperpigmented papules and a distinct histopathology which demonstrates pigmented filiform and branching rete pegs originating at the follicular infundibulum with many epidermal horn cysts while the interfollicular epidermis is essentially normal. Hereby, we present a case of follicular DDD with hidradenitis suppurativa (HS). A 37-year-old Thai man presented with slowly progressive hyperpigmented comedone-like papules on the face, neck, axillae, upper trunk, and buttocks with perioral pitted scars. Punch biopsy from a comedonal lesion on his back was consistent with follicular DDD. He also had recurrent painful nodules and abscess on the back, groin, and buttock which matched the clinical criteria for the diagnosis of HS. To date, a paucity of concurrent DDD with HS has been reported. Recent genetic studies speculate a shared pathophysiologic mechanism of DDD and HS.
\end{abstract}




\section{Introduction}

Dowling-Degos disease (DDD) is a progressive reticulate pigmentary disorder first reported by Dowling and Freudenthal [1] and Degos and Ossipowski [2], respectively. It usually is inherited in an autosomal dominant pattern; however, some cases are sporadic [3]. The main genetic mutation associated with DDD is KRT5. Keratin 5 is an element in the cytoskeleton of basal keratinocytes. To date, many genetic mutations have been documented such as POFUT1, POGLUT1, and PSENEN [3]. DDD has multiple variants of DDD including classic DDD, generalized DDD, Galli-Galli disease, Haber's syndrome, pigmentatio reticularis faciei et colli, and follicular DDD [4]. According to literature reviews, DDD has been found to be associated with severe nodulocystic acne, hidradenitis suppurativa (HS), chloracne-like lesions, seborrheic keratoses, dystrophic fingernails, and an accessory tragus [3,5]. DDD is known to be resistant to treatment, and no standard treatment has been established [3].

\section{Case Report}

A 37-year-old Thai man with an underlying disease of hepatitis C cirrhosis presented with slowly progressive hyperpigmented comedone-like papules on the face, neck, axillae, upper trunk, groin, and buttocks with perioral pitted scars and numerous atrophic scars. The skin lesions first appeared 22 years ago. They were mostly asymptomatic, so he did not seek any treatment. There were a few times when he experienced some painful nodules and abscess on his back, groin, and buttock which healed with atrophic scars. On one occasion, he had a tender nodule with pus around his anus which later on was diagnosed as perianal abscess. He received oral antibiotics and did not require surgery. He denied anyone in his family having similar symptoms.

Upon examination, he had numerous hyperpigmented macules and papules with numerous open comedones and atrophic scars on the face, neck, chest, axillae, upper back, groin, and buttocks with few painful nodules on his back, groin, and buttock (shown in Fig. 1). No pus, discharge, or sinus tracts were found. Other mucosa, palms, soles, hair, and nails were normal.

Histopathology from a comedonal lesion at the back revealed dilated follicular infundibulum filled with keratin with filiform-like rete ridges and increased basal melanin pigmentation involving follicular area while interfollicular epithelium remains normal (shown in Fig. 2). Considering the history, clinical presentation, and histopathology, this patient was diagnosed with follicular DDD with HS. In terms of treatment, he received anti-acne lotion which is a mixture of $1 \%$ resorcinol, $1 \%$ salicylic acid, and $6.7 \%$ zinc oxide. The patient did not come for follow-up.

\section{Discussion}

DDD is an uncommon autosomal dominant genodermatosis affecting individuals during the third to fourth decade of life with progressive hyperpigmented macules and papules with hyperkeratosis [6]. Lesions initially appear on axillae and groins followed by intergluteal, inframammary folds, neck, trunk, and upper extremities. Most patients are asymptomatic, but few have pruritus on the affected area. At the present, several variations of clinical, histopathology, and genetic mutations have been described such as classic DDD, generalized DDD, follicular DDD, and Galli-Galli disease [3, 4].

Our patient presented with slowly progressive hyperpigmented macules and papules with numerous open comedones and atrophic scars on the face, neck, chest, axillae, upper back,

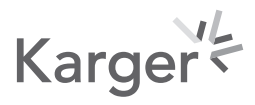




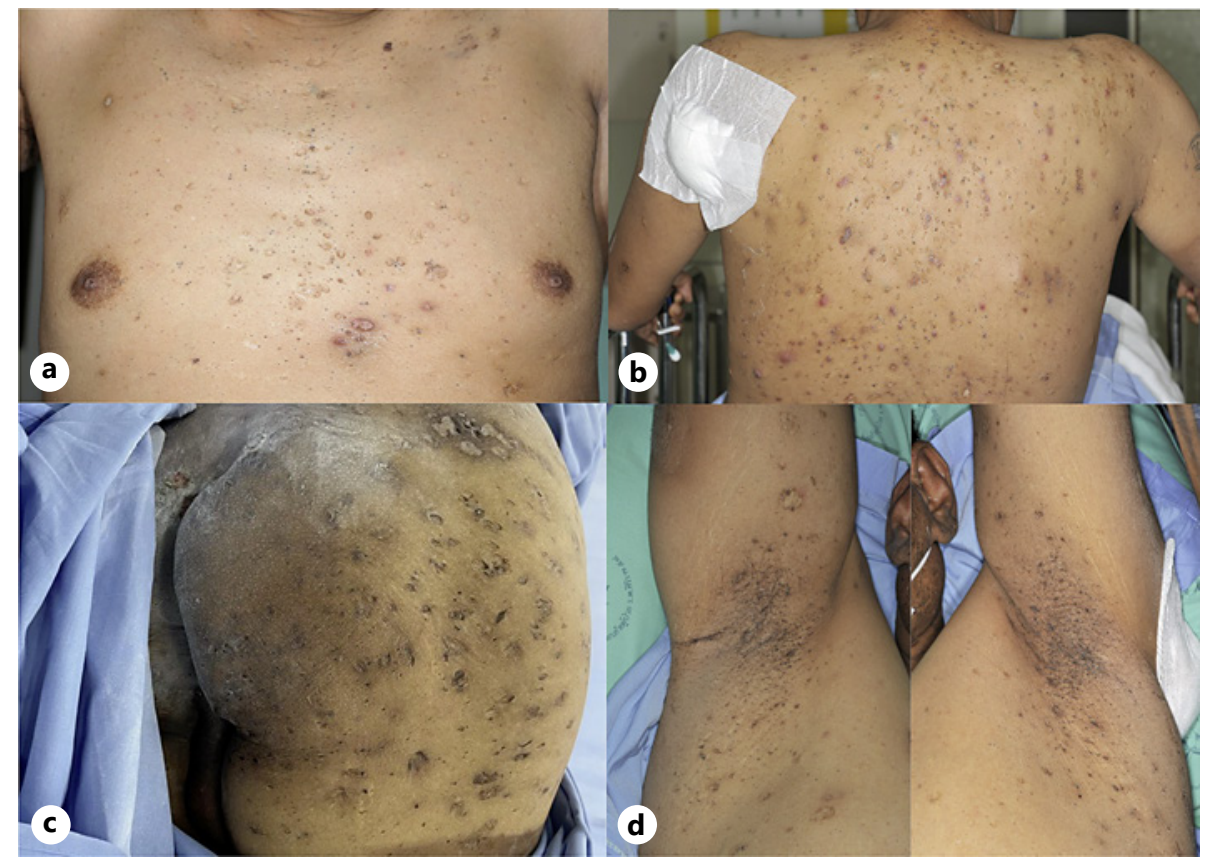

Fig. 1. The patient had numerous hyperpigmented macules and papules with numerous open comedones and atrophic scars on the chest (a) and few tender erythematous nodules on the back (b) and both buttocks (c). d He also had hyperpigmented macules on both axillae.

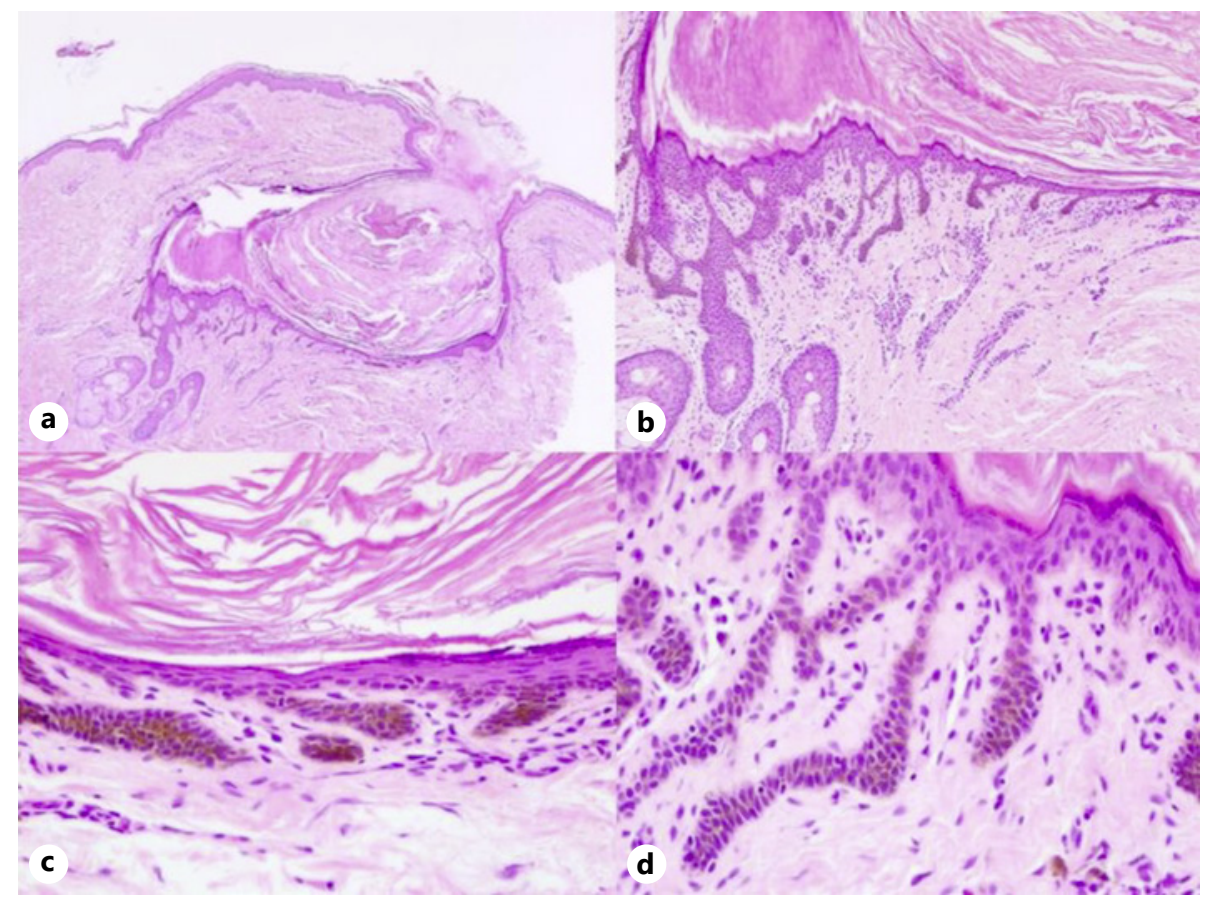

Fig. 2. a Histopathology from a comedonal lesion on the back at scanning power revealed dilated follicular infundibulum filled with keratin and filiform-like rete ridges involving follicular area while interfollicular epithelium remains normal. b, c, d Medium- and high-power examination showed filiform-like rete ridges with basal hyperpigmentation. 
Table 1. Case reports of follicular Dowling-Degos disease

\begin{tabular}{llllll}
\hline Case no. [Refs.] & Sex & Age of onset, years & Age, years & Family history & Associated disease \\
\hline $1[5]$ & Male & 10 & 25 & Yes & Ichthyosis vulgaris \\
$2[5]$ & Female & 5 & 19 & Yes & n/a \\
$3[4]$ & Female & Puberty & 32 & Yes & n/a \\
$4[4]$ & Female & Puberty & 12 & Yes & n/a \\
$5[9]$ & Female & 23 & 35 & Yes & n/a \\
$6[9]$ & Female & 27 & 43 & Yes & n/a \\
$7[9]$ & Male & 36 & 54 & Yes & n/a \\
$8[10]$ & Female & 15 & 30 & Yes & Hidradenitis suppurativa \\
$9[11]$ & Female & Childhood & 29 & No & Blepharophimosis-ptosis- \\
& & & & & epicanthus inversus \\
$10[12]$ & Male & Childhood & 28 & Yes & syndrome \\
$11[12]$ & Male & Childhood & 23 & Yes & Lichen planus \\
12 (our case) & Male & 15 & 37 & No & Hidradenitis suppurativa \\
\hline
\end{tabular}

n/a, not available.

and buttock since he was 15 years old. Differential diagnosis at first included HS and familial dyskeratotic comedones. Familial dyskeratotic comedones present at puberty with numerous, discrete, disseminate, open comedones that can heal with scar on the trunk, arms, leg, and shaft of the penis, sparing the palms and soles [7]. These features seem similar to our patient. However, in familial dyskeratotic comedones, the histopathology only shows dyskeratosis and invaginations into the dermis with occasional acantholysis which is not seen in our patient. Therefore, this patient was diagnosed with follicular DDD. Moreover, this patient also had recurrent painful nodules and abscess with multiple atrophic scars on the back, buttock, and perianal area which is not found in DDD alone. These clinical features were consistent with all 3 criteria of HS including one or more typical lesion, typical distribution, and recurrence of symptoms [8]. Typical lesions of HS are painful nodules, abscess, sinus tracts, double-open comedones, and bridges scars. Therefore, we concluded that our patient had concurrent DDD and HS.

To our knowledge, 12 cases (including our case) have been reported so far (shown in Table 1) [4, 5, 9-12]. All cases had clinical and histopathology characteristic of follicular DDD. Dermoscopic examination demonstrated brown pigmented Chinese letter pattern or irregular star shape, central brown hyperkeratotic follicular plugs, and comedones [9]. However, half of the reported cases did not have hyperpigmented macules and papules on flexural areas. Interestingly, most cases of follicular DDD present around childhood and puberty while classic DDD commonly presents after puberty. Additional features include ichthyosis vulgaris, lichen planus, HS, and blepharophimosis-ptosis-epicanthus inversus syndrome [5, 10-12].

HS is a chronic inflammatory disorder of the hair and follicles typically involving axillae, buttocks, groin, perineal, and inframammary regions [8]. According to case reports and clinical studies, concurrent DDD with HS has been reported in 53 cases (including our case) as shown in Table 2 [10,13-33]: 2 cases of which are follicular DDD variant and 1 case is associated with Galli-Galli disease, a variant of DDD [10,33]. Most cases reported were of female predominance and more than half presented with at least Hurley stage 2 . Almost all had a family history of HS. Recent genetic studies have found that patients with concurrent DDD with HS mostly have mutation in PSENEN, followed by POFUT1 and a case of NCSTN $[13,14,25,27,28,31]$. 
Table 2. Reported cases and clinical studies of concurrent Dowling-Degos disease with hidradenitis suppurativa

\begin{tabular}{|c|c|c|c|c|c|c|}
\hline Case no. [Refs.] & Sex & Age, years & $\begin{array}{l}\text { Family } \\
\text { history } \\
\text { of DDD }\end{array}$ & $\begin{array}{l}\text { Family } \\
\text { history } \\
\text { of HS }\end{array}$ & $\begin{array}{l}\text { Genetic } \\
\text { mutation }\end{array}$ & $\begin{array}{l}\text { Associated } \\
\text { disease }\end{array}$ \\
\hline $1-15[24]$ & 8 female/7 male & $43.2 \pm 12.36$ & 9 yes $/ 6$ no & Yes & $\mathrm{n} / \mathrm{a}$ & $\mathrm{n} / \mathrm{a}$ \\
\hline $16-20[25]$ & 3 female $/ 2$ male & $\mathrm{n} / \mathrm{a}$ & $\mathrm{n} / \mathrm{a}$ & $\mathrm{n} / \mathrm{a}$ & PSENEN & $\mathrm{n} / \mathrm{a}$ \\
\hline $21[26]$ & Male & 47 & No & Yes & $\mathrm{n} / \mathrm{a}$ & $\mathrm{n} / \mathrm{a}$ \\
\hline 22 [27] & Male & 54 & No & Yes & NCSTN & $\mathrm{n} / \mathrm{a}$ \\
\hline $23-26$ [14] & 3 female $/ 1$ male & $\mathrm{n} / \mathrm{a}$ & Yes & Yes & PSENEN & $\mathrm{n} / \mathrm{a}$ \\
\hline 27 [13] & Female & 33 & $\mathrm{n} / \mathrm{a}$ & $\mathrm{n} / \mathrm{a}$ & POFUT1 & $\mathrm{n} / \mathrm{a}$ \\
\hline $28[28]$ & Male & 31 & Yes & Yes & PSENEN & $\mathrm{n} / \mathrm{a}$ \\
\hline $29-30$ [29] & 2 female & $39 / 45$ & Yes & Yes & $\mathrm{n} / \mathrm{a}$ & $\mathrm{n} / \mathrm{a}$ \\
\hline $31[30]$ & Female & 40 & Yes & Yes & $\mathrm{n} / \mathrm{a}$ & Arthritis \\
\hline 32 [31] & Female & 34 & Yes & Yes & POFUT1 & $\mathrm{n} / \mathrm{a}$ \\
\hline 33 [32] & Female & 38 & Yes & Yes & $\mathrm{n} / \mathrm{a}$ & $\mathrm{n} / \mathrm{a}$ \\
\hline $34^{\mathrm{a}}[10]$ & Female & 30 & Yes & No & $\mathrm{n} / \mathrm{a}$ & $\mathrm{n} / \mathrm{a}$ \\
\hline 35 [15] & Female & 43 & Yes & Yes & $\mathrm{n} / \mathrm{a}$ & $\mathrm{n} / \mathrm{a}$ \\
\hline $36[16]$ & Female & 44 & Yes & Yes & $\mathrm{n} / \mathrm{a}$ & $\mathrm{n} / \mathrm{a}$ \\
\hline $37-38$ [17] & 2 female & $23 / 48$ & Yes & Yes & $\mathrm{n} / \mathrm{a}$ & Arthritis \\
\hline $39[18]$ & Female & 49 & Yes & Yes & $\mathrm{n} / \mathrm{a}$ & $\mathrm{n} / \mathrm{a}$ \\
\hline 40 [19] & Male & 68 & No & Yes & $\mathrm{n} / \mathrm{a}$ & SCC \\
\hline $41^{\mathrm{b}}[33]$ & Female & 33 & No & No & $\mathrm{n} / \mathrm{a}$ & $\mathrm{n} / \mathrm{a}$ \\
\hline $42[23]$ & $\mathrm{n} / \mathrm{a}$ & $\mathrm{n} / \mathrm{a}$ & $\mathrm{n} / \mathrm{a}$ & $\mathrm{n} / \mathrm{a}$ & $\mathrm{n} / \mathrm{a}$ & SCC \\
\hline $43[20]$ & Female & 39 & $\mathrm{n} / \mathrm{a}$ & $\mathrm{n} / \mathrm{a}$ & $\mathrm{n} / \mathrm{a}$ & $\mathrm{n} / \mathrm{a}$ \\
\hline $44[22]$ & Female & 38 & $\mathrm{n} / \mathrm{a}$ & $\mathrm{n} / \mathrm{a}$ & $\mathrm{n} / \mathrm{a}$ & KA \\
\hline $45-52[21]$ & $\mathrm{n} / \mathrm{a}$ & $\mathrm{n} / \mathrm{a}$ & $\mathrm{n} / \mathrm{a}$ & $\mathrm{n} / \mathrm{a}$ & $\mathrm{n} / \mathrm{a}$ & $\mathrm{n} / \mathrm{a}$ \\
\hline $53^{\mathrm{a}}$ (Our case) & Male & 37 & No & No & $\mathrm{n} / \mathrm{a}$ & $\mathrm{n} / \mathrm{a}$ \\
\hline
\end{tabular}

Few cases reported association with squamous cell carcinoma, keratoacanthoma, and arthritis $[17,19,22,23,30]$.

In 2017, Pavolsky et al. [14] discovered that patients who presented with features of both DDD and HS have heterozygous mutation in PSENEN. A year later, González-Villanueva et al. [13] found that POFUT1 mutation is associated with combined DDD and HS as well. Lately, NCSTN mutation was found in a concurrent DDD with HS patient [27]. All PSENEN and POFUT1 are critical genes that regulate the Notch signaling pathway and have been speculated to be the pathophysiologic mechanisms of patients with combined DDD and HS [3]. However, NCSTN may not have a direct effect on the NOTCH pathway but is believed to stimulate inflammatory response via type I interferon gene expression [27].

As other variants of DDD, follicular DDD is also resistant to treatment. Oral isotretinoin has been prescribed in many cases and had shown to reduce follicular lesions and comedones. However, when discontinued, follicular DDD relapses [9]. Other treatments include topical hydroquinone, retinoids, and corticosteroids [6]. 
In conclusion, follicular DDD, a new variant of DDD, is diagnosed based on clinical characteristics and distinct histopathology. Concurrent DDD with HS was reported increasingly. Further studies are needed to clearly explain the pathophysiology and genetic mutation involved.

\section{Statement of Ethics}

Written informed consent was obtained from the patient for publication of this case report and any accompanying images. The study has been done according to the Declaration of Helsinki. Ethical approval was not required for this study in accordance with national guidelines.

\section{Conflict of Interest Statement}

The authors have no conflicts of interest to declare.

\section{Funding Sources}

No funding was received.

\section{Author Contributions}

All authors participated in writing and are fully responsible for the manuscript.

\section{Data Availability Statement}

All data supporting this study are available from Dr. Charussri Leeyaphan upon request.

\section{References}

Dowling GB, Freudenthal W. Acanthosis nigricans. Proc R Soc Med. 1938 Jul;31(9):1147-50.

2 Degos R, Ossipowski B. Reticulated pigmentary dermatosis of the folds: relation to acanthosis nigricans. Ann Dermatol Syphiligr. 1954 Mar-Apr;81(2):147-51.

3 Stephan C, Kurban M, Abbas O. Dowling-Degos disease: a review. Int J Dermatol. 2021 Aug;60(8):944-50.

4 Mahajan SH, Mahajan SA, Khopkar US, Kharkar VD. Follicular Dowling-Degos disease: a rare pigmentary dermatosis. Indian Dermatol Online J. 2017 Nov-Dec;8(6):487-9.

5 Singh S, Khandpur S, Verma P, Singh M. Follicular Dowling Degos disease: a rare variant of an evolving dermatosis. Indian J Dermatol Venereol Leprol. 2013 Nov-Dec;79(6):802-4.

6 Chang MW. Disorders of hyperpigmentation. In: Bolognia JL, Schaffer JV, Cerroni L, editors. Dermatology. 4th ed Philadelphia: Elsevier; 2018. p. 1138-9.

7 Kumaran MS, Appachu D, Jayaseelan E. Familial dyskeratotic comedones. Indian J Dermatol Venereol Leprol. 2008 Mar-Apr;74(2):142-4.

8 Okoye GA. Hidradenitis suppurativa. In: Kang S, Amagai M, Bruckner AL, Enk AH, Margolis DJ, McMichael AJ, editors, et al. Fitzpatrick's dermatology. 9th ed New York: McGraw Hill; 2019. p. 1484.

9 Dabas G, Mahajan R, Afra TP, De D, Handa S, Aggarwal D, et al. Dermoscopy of follicular Dowling-Degos disease. Indian J Dermatol. 2020 Jul-Aug;65(4):290-4.

10 Supekar BB, Rambhia KD, Singh RP, Mukhi JI. Dowling-Degos disease with follicular involvement associated with hidradenitis suppurativa: a manifestation of follicular occlusion phenomenon? Indian J Dermatol. 2020 Jul-Aug;65(4):295-8. 
Nokdhes et al.: Follicular DDD with HS

11 Kumari P, Suvirya S, Singhai A, Malhotra K, Pathania S. Follicular Dowling-Degos disease and blepharophimosisptosis-epicanthus inversus syndrome: a chance or significant association. Indian J Dermatol. 2021 Jan-Feb; 66(1):100-1.

12 Agarwal P, Jagati A, Neazee S, Patel SV. Follicular Dowling Degos disease with lichen planus in two siblings: a rare association. Indian Dermatol Online J. 2021 May-Jun 12;12(3):459-61.

13 González-Villanueva I, Gutiérrez M, Hispán P, Betlloch I, Pascual JC. Novel POFUT1 mutation associated with hidradenitis suppurativa-Dowling-Degos disease firm up a role for Notch signalling in the pathogenesis of this disorder. Br J Dermatol. 2018 Apr;178(4):984-6.

14 Pavlovsky M, Sarig O, Eskin-Schwartz M, Malchin N, Bochner R, Mohamad J, et al. A phenotype combining hidradenitis suppurativa with Dowling-Degos disease caused by a founder mutation in PSENEN. Br J Dermatol. 2018 Feb;178(2):502-8.

15 Arjona-Aguilera C, Linares-Barrios M, Albarrán-Planelles C, Jiménez-Gallo D. Dowling-Degos disease associated with hidradenitis suppurativa: a case report. Actas Dermosifiliogr. 2015 May;106(4):337-8.

16 Choudhary SV, Jain D, Agrawal P, Singh A. Dowling-Degos disease and hidradenitis suppurativa: co-occurrence or association? Indian Dermatol Online J. 2013 Jul;4(3):191-4.

17 Dixit R, George R, Jacob M, Sudarsanam TD, Danda D. Dowling-Degos disease, hidradenitis suppurativa and arthritis in mother and daughter. Clin Exp Dermatol. 2006 May;31(3):454-6.

18 Loo WJ, Rytina E, Todd PM. Hidradenitis suppurativa, Dowling-Degos and multiple epidermal cysts: a new follicular occlusion triad. Clin Exp Dermatol. 2004 Nov;29(6):622-4.

19 Li M, Hunt MJ, Commens CA. Hidradenitis suppurativa, Dowling Degos disease and perianal squamous cell carcinoma. Australas J Dermatol. 1997 Nov;38(4):209-11.

20 Bedlow AJ, Mortimer PS. Dowling-Degos disease associated with hidradenitis suppurativa. Clin Exp Dermatol. 1996 Jul;21(4):305-6.

21 Balus L, Fazio M, Amantea A, Menaguale G. [Dowling-Degos disease and Verneuil disease]. Ann Dermatol Venereol. 1993;120(10):705-8.

22 Fenske NA, Groover CE, Lober CW, Espinoza CG. Dowling-Degos disease, hidradenitis suppurativa, and multiple keratoacanthomas. A disorder that may be caused by a single underlying defect in pilosebaceous epithelial proliferation. J Am Acad Dermatol. 1991 May;24(5):888-92.

23 Weber LA, Kantor GR, Bergfeld WF. Reticulate pigmented anomaly of the flexures (Dowling-Degos disease): a case report associated with hidradenitis suppurativa and squamous cell carcinoma. Cutis. 1990 Jun;45(6): 446-50.

24 Agut-Busquet E, González-Villanueva I, Romani de Gabriel J, Pascual JC, Ribera Pibernat M, Luelmo J, et al. Dowling-Degos disease and hidradenitis suppurativa. Epidemiological and clinical study of 15 patients and review of the literature. Acta Derm Venereol. 2019 Sep 1;99(10):917-8.

25 Ralser DJ, Basmanav FB, Tafazzoli A, Wititsuwannakul J, Delker S, Danda S, et al. Mutations in $\gamma$-secretase subunit-encoding PSENEN underlie Dowling-Degos disease associated with acne inversa. J Clin Invest. 2017 Apr 3;127(4):1485-90.

26 Kleeman D, Trüeb RM, Schmid-Grendelmeier P. [Reticular pigmented anomaly of the flexures. Dowling-Degos disease of the intertrigo type in association with acne inversa]. Hautarzt. 2001 Jul;52(7):642-5.

27 Garcovich S, Tricarico PM, Nait-Meddour C, Giovanardi G, Peris K, Crovella S, et al. Novel nicastrin mutation in hidradenitis suppurativa-Dowling-Degos disease clinical phenotype: more than just clinical overlap? Br J Dermatol. 2020 Oct;183(4):758-9.

28 Peter DCV, Smith FJD, Wilson NJ, Danda S. PSENEN mutation in coexistent hidradenitis suppurativa and Dowling-Degos disease. Indian Dermatol Online J. 2021 Jan-Feb;12(1):147-9.

29 Cortez Cardoso Penha R, Cortez de Almeida RF, Câmara Mariz J, Brewer Lisboa L, do Nascimento Barbosa L, Souto da Silva R. The deregulation of NOTCH pathway, inflammatory cytokines, and keratinization genes in two Dowling-Degos disease patients with hidradenitis suppurativa. Am J Med Genet A. 2020 Nov; 182(11): 2662-5.

30 Rammeh Rommani S, Ajouli W, Fazaa B, Khaled A, Zermani R. [Dowling-Degos disease and chronic hidradenitis suppurativa]. Ann Dermatol Venereol. 2013 Dec;140(12):838-40.

31 García-Gil MF, Monte Serrano J, Ramirez-Lluch M, Valero Torres A, López-Giménez MT, Lezcano Biosca V. A novel mutation in POFUT1 gene associated with Dowling-Degos disease and hidradenitis suppurativa. Int J Dermatol. 2021 Jan;60(1):e25-7.

32 George A, George R, Mathew AJ, Telugu RB. Dowling-Degos disease with hidradenitis suppurativa and inflammatory arthritis in two generations. Indian Dermatol Online J. 2020 May 10;11(3):413-5.

33 Del Mar M, González M, Sayed C, Phadke P. Hidradenitis suppurativa associated with Galli-Galli disease: extending the link with Dowling-Degos disease. J Clin Aesthet Dermatol. 2020 Dec;13(12):38-40. 